\title{
Countable partitions of the sets of points and lines
}

\author{
by
}

\author{
James H. S c h mer l (Storrs, Conn.)
}

\begin{abstract}
The following theorem is proved, answering a question raised by Davies in 1963. If $L_{0} \cup L_{1} \cup L_{2} \cup \ldots$ is a partition of the set of lines of $\mathbb{R}^{n}$, then there is a partition $\mathbb{R}^{n}=S_{0} \cup S_{1} \cup S_{2} \cup \ldots$ such that $\left|\ell \cap S_{i}\right| \leq 2$ whenever $\ell \in L_{i}$. There are generalizations to some other, higher-dimensional subspaces, improving recent results of Erdős, Jackson \& Mauldin.
\end{abstract}

0. Introduction. A series of papers, beginning in 1919 with Sierpiński [7] and ending with the 1963 paper of Davies [1], culminates in the following theorem. Simms [8] presents a detailed and fascinating account of the history of this and related theorems.

TheOREM 0.1 (Davies). If $m<\omega$, then the following are equivalent:

(1) $2^{\aleph_{0}} \leq \aleph_{m}$

(2) if $2 \leq n<\omega$ and $L=L_{0} \cup L_{1} \cup \ldots \cup L_{m+1}$ is a partition of the set of lines of $\mathbb{R}^{n}$, then there is a partition $\mathbb{R}^{n}=S_{0} \cup S_{1} \cup \ldots \cup S_{m+1}$ such that for each $\ell \in L_{i}, \ell \cap S_{i}$ is finite;

(3) there are pairwise nonparallel lines $d_{0}, d_{1}, \ldots, d_{m+1}$ in $\mathbb{R}^{2}$ and a partition $\mathbb{R}^{2}=S_{0} \cup S_{1} \cup \ldots \cup S_{m}$ such that if a line $\ell$ is parallel to $d_{i}$, then $\ell \cap S_{i}$ is finite.

Prior to the publication of this result, yet motivated by some similar results on finite partitions, Erdős [3] had asked about infinite partitions: If $L=L_{0} \cup L_{1} \cup L_{2} \cup \ldots$ is a countable partition of the set of lines of $\mathbb{R}^{2}$, is there a partition $\mathbb{R}^{2}=S_{0} \cup S_{1} \cup S_{2} \cup \ldots$ such that for each $\ell \in L_{i},\left|\ell \cap S_{i}\right| \leq 1$ ? This question was answered negatively by Davies [2]. However, on the positive side, Davies [2] proved the following two closely related theorems.

1991 Mathematics Subject Classification: 03E05, 04A20.

Key words and phrases: infinite partitions, Euclidean space.

Thanks to Dan Mauldin and Steve Jackson who spotted an error in a preliminary version of this paper. 
TheOREM 0.2. Let $L=L_{0} \cup L_{1} \cup L_{2} \cup$. . be a countable partition of the set of lines of $\mathbb{R}^{n}$. Then there is a countable partition $\mathbb{R}^{n}=S_{0} \cup S_{1} \cup S_{2} \cup \ldots$ such that whenever $\ell \in L_{i}$, then $\ell \cap S_{i}$ is finite.

TheOREM 0.3. (Assume $2^{\aleph_{0}} \leq \aleph_{m}$.) Let $L=L_{0} \cup L_{1} \cup L_{2} \cup \ldots$ be a countable partition of the set of lines of $\mathbb{R}^{n}$. Then there is a countable partition $\mathbb{R}^{n}=S_{0} \cup S_{1} \cup S_{2} \cup \ldots$ such that whenever $\ell \in L_{i}$, then $\left|\ell \cap S_{i}\right| \leq$ $m+1$.

Some generalizations of Theorems 0.2 and 0.3 can be found in Erdös, Jackson \& Mauldin [4]. Influenced by $(2) \Rightarrow(1)$ of Theorem 0.1, Davies [2] suggested that the converse of Theorem 0.3 might be true (more precisely: that ZFC proves that the statement in Theorem 0.3 implies that $2^{\aleph_{0}} \leq \aleph_{m}$ ), and in [4] it is asked if it is true (or consistent) that the conclusion of Theorem 0.2 can be strengthened to $\left|\ell \cap S_{i}\right| \leq 2$, perhaps for just $n=2$. It was then subsequently proved by Erdős, Jackson \& Mauldin [5] that it is consistent that the converse of Theorem 0.3 does not hold for $n=2$ and $m \geq 2$; specifically, it was proved that Martin's Axiom (MA) implies that the conclusion in Theorem 0.2 could be replaced by $\left|\ell \cap S_{i}\right| \leq 3$. However, this still left open the possibility that the converse of Theorem 0.3 held when $m=1$. These questions, and similar ones, are answered in this paper, the main result of which is the following definitive improvement to Theorems 0.2 and 0.3 .

THEOREM 0.4. Let $L=L_{0} \cup L_{1} \cup L_{2} \cup \ldots$ be a countable partition of the set of lines of $\mathbb{R}^{n}$. Then there is a countable partition $\mathbb{R}^{n}=S_{0} \cup S_{1} \cup S_{2} \cup \ldots$ of the set of points such that whenever $\ell \in L_{i}$, then $\left|\ell \cap S_{i}\right| \leq 2$.

A proof of this theorem and some other theorems on partitions of the sets of points and lines will be given in $\S 2$, and in $\S 3$ there are some generalizations concerning partitions of the sets of points and hyperplanes. The method of proof of Theorem 0.4 is derived from [6] where some similar sorts of theorems are proved. In $\S 1$ we review some preliminary material from [6] and, at the same time, establish some conventions, terminology and notation.

1. Preliminaries. If $X$ is any set and $n<\omega$, then $[X]^{n}$ is the set of $n$-element subsets of $X$ and $[X]^{<\omega}$ is the set of finite subsets of $X$. We let $\sigma_{0}, \sigma_{1}, \sigma_{2}, \ldots$ be a fixed enumeration of all finite sequences of elements of $\mathbb{Q}$, the set of rationals.

If $A \in[\mathbb{R}]^{<\omega}$ and we write $A=\left\{a_{0}, a_{1}, \ldots, a_{n-1}\right\}_{<}$, then it is to be understood that $a_{0}<a_{1}<\ldots<a_{n-1}$. We say that a set $\left\{r_{0}, r_{1}, \ldots, r_{n}\right\}_{<}$ of rationals is a rational separator for $A$ if

$$
r_{0}<a_{0}<r_{1}<a_{1}<r_{2}<\ldots<r_{n-1}<a_{n-1}<r_{n} .
$$


We will think of $\mathbb{R}$ as an ordered field. Let $A \subseteq \mathbb{R}$. If $D \subseteq \mathbb{R}^{k}$ is definable by a first-order formula in the language of ordered fields with parameters from $A$, then we say that $D$ is $A$-definable. If $D$ is $\emptyset$-definable, then it is definable. Let $\varphi_{0}, \varphi_{1}, \varphi_{2}, \ldots$ be a fixed enumeration of all definable analytic functions $\varphi: U \rightarrow \mathbb{R}^{n}$, where $k, n<\omega$ and $U \subseteq \mathbb{R}^{k}$ is a definable open set.

Let $T \subseteq \mathbb{R}$ be some fixed transcendence basis for $\mathbb{R}$. If $t \in T$, then $\{t\}$ is not $(T \backslash\{t\})$-definable. For each $a \in \mathbb{R}^{n},\{a\}$ is $T$-definable; moreover, there is a unique smallest (finite) subset of $T$, which we call the support of $a$ and denote by $\operatorname{supp}(a)$, such that $\{a\}$ is $\operatorname{supp}(a)$-definable. If $a \in \mathbb{R}^{n}$ and $\operatorname{supp}(a)=\left\{t_{0}, t_{1}, \ldots, t_{k-1}\right\}_{<}$, then there is a definable analytic function $\varphi\left(x_{0}, x_{1}, \ldots, x_{k-1}\right)$ mapping some open neighborhood $U$ of $\left\langle t_{0}, t_{1}, \ldots, t_{k-1}\right\rangle$ into $\mathbb{R}^{n}$ such that $a=\varphi\left(t_{0}, t_{1}, \ldots, t_{k-1}\right)$. We call the function $\varphi_{j}$ the defining function for $a$ if $j$ is the least such that $a=\varphi_{j}\left(t_{0}, t_{1}, \ldots, t_{k-1}\right)$. For such a function $\varphi_{j}$, all the partial derivatives at $\left\langle t_{0}, t_{1}, \ldots, t_{k-1}\right\rangle$ are nonzero. We call the function $\theta(x)=\varphi_{j}\left(t_{0}, t_{1}, \ldots, t_{p-1}, x, t_{p+1}, \ldots, t_{k-1}\right)$ the $p$ th coordinate function for $a$. Notice that the $p$ th coordinate function is $\left\{t_{0}, t_{1}, \ldots, t_{p-1}, t_{p+1}, \ldots, t_{k-1}\right\}$-definable. We refer to the line which contains $a$ and is parallel to $\theta^{\prime}\left(t_{p}\right)$ as the $p$ th tangent line at $a$.

If $V$ is a vector space over $\mathbb{R}$ and $X \subseteq V$, then the span of $X$, denoted by $\operatorname{Span}(X)$, is the smallest subspace of $V$ containing $X$. A line of $V$ is a translate of a 1-dimensional subspace. Let $\mathcal{L}(V)$ be the set of lines of $V$.

2. Points and lines. Theorem 0.4 is a consequence of the following theorem, which will have some other consequences as well. Notice that Theorem 0.4 concerns $\mathbb{R}^{n}$ as an $n$-dimensional vector space over $\mathbb{R}$, whereas Theorem 2.1 concerns some infinite-dimensional vector spaces. Nothing would be lost if in Theorem 2.1 we considered some specific $2^{\aleph_{0}}$-dimensional vector space, such as a separable real Hilbert space or the space $\mathbb{R}^{\omega}$ of all real sequences.

THeOREM 2.1. Let $V$ be a vector space over $\mathbb{R}$ such that $\operatorname{dim}(V) \leq 2^{\aleph_{0}}$. Then there are functions $\pi: V \rightarrow \omega$ and $\lambda: V \rightarrow[\mathcal{L}(V)]^{<\omega}$ such that whenever $\ell \in \mathcal{L}(V), a, b, c \in \ell$ are distinct and $\pi(a)=\pi(b)=\pi(c)$, then $\ell \in \lambda(a) \cap \lambda(b) \cap \lambda(c)$.

Before proving Theorem 2.1, we show how it implies Theorem 0.4.

Proof of Theorem 0.4. To deduce Theorem 0.4 from Theorem 2.1, we will need the latter theorem only in the case where $V=\mathbb{R}^{n}$. Let $\pi$ and $\lambda$ be as in Theorem 2.1. Given $a \in \mathbb{R}^{n}$, we will determine how to color $a$; that is, we will determine some $i<\omega$ and then declare that $a \in S_{i}$.

Let $i, s<\omega$ be such that $\sigma_{i}=\langle\pi(a), s\rangle$ and $\lambda(a) \cap L_{i}=\emptyset$. There are such $i, s$ since $\lambda(a)$ is finite. Now let $a \in S_{i}$. 
The partition $\mathbb{R}^{n}=S_{0} \cup S_{1} \cup S_{2} \cup \ldots$ has just been defined. We will show that it meets the requirements. For a contradiction, suppose that $a, b, c \in$ $\ell \cap S_{i}$ are distinct and $\ell \in L_{i}$. Let $\sigma_{i}=\langle r, s\rangle$. Thus, $\pi(a)=\pi(b)=\pi(c)=r$, so that $\ell \in \lambda(a) \cap L_{i}$, a contradiction.

It would have sufficed in proving Theorem 0.4 from Theorem 2.1 if Theorem 2.1 had the weaker conclusion that $\ell \in \lambda(a) \cup \lambda(b) \cup \lambda(c)$. This comment is relevant in proving Corollary 2.5.

Proof of Theorem 2.1. The theorem will be proved first for finite-dimensional $V$, and then for infinite-dimensional $V$.

Suppose $V$ is a finite-dimensional vector space over $\mathbb{R}$. We will assume that $V=\mathbb{R}^{n}$. For each $a \in \mathbb{R}^{n}$ we will determine $\pi(a)$ and $\lambda(a)$.

Let $\operatorname{supp}(a)=\left\{t_{0}, t_{1}, \ldots, t_{k-1}\right\}_{<}$, and let $\left\{r_{0}, r_{1}, \ldots, r_{k}\right\}_{<}$be a rational separator for $\operatorname{supp}(a)$. Let $\varphi_{j}: U \rightarrow \mathbb{R}^{n}$ be the defining function for $a$. Then let $\lambda(a)$ be the set of tangent lines at $a$, and let $\pi(a)=i$, where $\sigma_{i}=\left\langle r_{0}, r_{1}, \ldots, r_{k}, j\right\rangle$.

The functions $\pi$ and $\lambda$ have been defined. We now show that they have the required property. So, suppose that $\ell$ is a line, $a, b, c \in \ell$ are distinct and $i=\pi(a)=\pi(b)=\pi(c)$. Let $\sigma_{i}=\left\langle r_{0}, r_{1}, \ldots, r_{k}, j\right\rangle$. Since $a, b$ are distinct there are $p<k, u \in \operatorname{supp}(a)$ and $v \in \operatorname{supp}(b)$ such that $r_{p}<u, v<r_{p+1}$ and $u \neq v$. Let $w \in \operatorname{supp}(c)$ be such that $r_{p}<w<r_{p+1}$. Without loss of generality, we can assume that $u \notin\{v, w\}$. Thus, $u \notin \operatorname{supp}(b) \cup \operatorname{supp}(c)$.

We now show that $\ell \in \lambda(a)$; in fact, letting $\ell_{p}$ be the $p$ th tangent line at $a$, we will show that $\ell=\ell_{p}$. Let $\theta(x)$ be the $p$ th coordinate function for $a$. For any integer $N>0$ there are $x$ such that $0<|x-u|<1$ and $\theta(x) \in \ell$, as otherwise $u$ would be $((\operatorname{supp}(a) \cup \operatorname{supp}(b) \cup \operatorname{supp}(c)) \backslash\{u\})$-definable, which is impossible. Clearly, then, $\ell=\ell_{p}$. Thus, $\ell \in \lambda(a)$. Notice that $\ell$ is $(\operatorname{supp}(a) \backslash\{u\})$-definable.

Having proved that $\ell \in \lambda(a)$, we can show that $\ell \in \lambda(b) \cap \lambda(c)$. Without loss of generality, consider $b$, and suppose $\ell \notin \lambda(b)$. Let $\ell^{\prime}$ be the $p$ th tangent line for $\varphi_{j}$ at $b$. Then, in particular, $\ell \neq \ell^{\prime}$, so $b$ is the unique point $x$ (in some definable open neighborhood of $b$ ) for which $\theta(x) \in \ell$. Then $u$ would be $((\operatorname{supp}(a) \cup \operatorname{supp}(b)) \backslash\{u\})$-definable, which is a contradiction. So $\ell \in \lambda(b)$.

Thus, $\ell \in \operatorname{supp}(a) \cap \operatorname{supp}(b) \cap \operatorname{supp}(c)$, which finishes the proof of the theorem in the case where $V$ is finite-dimensional. (As has already been mentioned, only the finite-dimensional case was needed to prove Theorem 0.4.)

Next we prove the theorem for infinite-dimensional $V$. Without loss of generality, assume that $\operatorname{dim}(V)=2^{\aleph_{0}}$, and let $\left\{v_{x}: x \in \mathbb{R}\right\}$ be a basis for $V$, where $x \neq y \Rightarrow v_{x} \neq v_{y}$. For each $a \in V$, let naps $(a)$ be the unique smallest finite subset $X \subseteq \mathbb{R}$ such that $a$ is in the span of $\left\{v_{x}: x \in X\right\}$. For each $X \in[\mathbb{R}]^{<\omega}$, let $V(X)$ be the finite-dimensional subspace of $V$ spanned by $\left\{v_{x}: x \in X\right\}$. By the first part of this proof, for each $X \in[\mathbb{R}]^{<\omega}$, let 
$\pi_{X}: V(X) \rightarrow \omega$ and $\lambda_{X}: V(X) \rightarrow[\mathcal{L}(V(X))]^{<\omega}$ satisfy the conclusion of the lemma.

We now define $\pi$ and $\lambda$. Consider $a \in V$ and let $X=\operatorname{naps}(a)$. Then set $\lambda(a)=\lambda_{X}(a)$. Let $X=\left\{x_{0}, x_{1}, \ldots, x_{k-1}\right\}_{<}$, let $\left\{r_{0}, r_{1}, \ldots, r_{k}\right\}_{<}$be a rational separator for $X$, and then let $\pi(a)=i$, where $\sigma_{i}=\left\langle r_{0}, r_{1}, \ldots, r_{k}, \pi_{X}(a)\right\rangle$.

The functions $\pi$ and $\lambda$ have been defined. We now show that they have the required property. So, suppose that $\ell \in \mathcal{L}(V)$ is a line, $a, b, c \in \ell$ are distinct and $i=\pi(a)=\pi(b)=\pi(c)$. Let $\sigma_{i}=\left\langle r_{0}, r_{1}, \ldots, r_{k}, j\right\rangle$. Since $a, b, c$ are collinear, it is clear that naps $(a)=\operatorname{naps}(b)=\operatorname{naps}(c)$, as otherwise it would be that, say, $\operatorname{naps}(a) \backslash(\operatorname{naps}(b) \cup \operatorname{naps}(c)) \neq \emptyset$, contradicting the collinearity. But then, letting $X=\operatorname{naps}(a)$, we have $j=\pi_{X}(a)=\pi_{X}(b)=$ $\pi_{X}(c)$, so that $a, b, c \in \ell^{\prime}$ for some $\ell^{\prime} \in \lambda_{X}(a) \cap \lambda_{X}(b) \cap \lambda_{X}(c)$. Clearly, $\ell^{\prime}=\ell \cap V(X)$, so that $a, b, c \in \ell \in \lambda(a) \cap \lambda(b) \cap \lambda(c)$.

The setup for Theorem 2.1 was more general than for Theorem 0.4. The same proof that deduced Theorem 0.4 from Theorem 2.1 can be used to get the correspondingly stronger result of the following corollary.

Corollary 2.2. Let $V$ be a vector space over $\mathbb{R}$ such that $\operatorname{dim}(V) \leq 2^{\aleph_{0}}$. Let $\mathcal{L}(V)=L_{0} \cup L_{1} \cup L_{2} \cup \ldots$ be a countable partition of the set of lines of $V$. Then there is a countable partition $V=S_{0} \cup S_{1} \cup S_{2} \cup \ldots$ such that whenever $\ell \in L_{i}$, then $\left|\ell \cap S_{i}\right| \leq 2$.

The next corollary is related to Theorem 0.4 (and more generally to Corollary 2.2). It is an improvement of Theorem 1.2 of [5] in four ways. The first is that we make no additional set-theoretic assumptions whereas MA was assumed in [5]. The second is that in [5] it was required that each line meet $S$ in a finite set, not a countable set. Third, the conclusion in [5] is that each line meets each $S_{i}$ in at most 3 points. And finally, Corollary 2.3 applies to some infinite-dimensional vector spaces.

Corollary 2.3. Let $V$ be a vector space over $\mathbb{R}$ such that $\operatorname{dim}(V) \leq 2^{\aleph_{0}}$. Let $S \subseteq V$ be such that each line of $V$ meets $S$ in a countable set. Then there is a countable partition $S=S_{0} \cup S_{1} \cup S_{2} \cup \ldots$ such that whenever $\ell$ is a line and $i<\omega$, then $\left|\ell \cap S_{i}\right| \leq 2$.

Proof. For each line $\ell$ which meets $S$, let $\left\{P_{j}(\ell): j<\omega\right\}$ be the set of points in $\ell \cap S_{i}$. For each $a \in V$ we will determine how to color $a$; that is, we will determine some $i<\omega$ and then declare that $a \in S_{i}$. We will use $\pi$ and $\lambda$ from Theorem 2.1. Let $e: \mathcal{L}(V) \rightarrow \mathbb{R}$ be an injection. Then let $\lambda(a)=$ $\left\{\ell_{0}, \ell_{1}, \ldots, \ell_{k-1}\right\}$, where $e\left(\ell_{0}\right)<e\left(\ell_{1}\right)<\ldots<e\left(\ell_{k}\right)$. Let $\left\{r_{0}, r_{1}, \ldots, r_{k}\right\}<$ be a rational separator for $e(\lambda(a))$, and let $j_{0}, j_{1}, \ldots, j_{k-1}<\omega$ be such that $a=P_{j_{0}}\left(\ell_{0}\right)=P_{j_{1}}\left(\ell_{1}\right)=\ldots=P_{j_{k-1}}\left(\ell_{k-1}\right)$. Then let

$$
\sigma_{i}=\left\langle r_{0}, r_{1}, \ldots, r_{k}, j_{0}, j_{1}, \ldots, j_{k-1}, \pi(a)\right\rangle .
$$

We now declare that $a \in S_{i}$. 
The partition $S=S_{0} \cup S_{1} \cup S_{2} \cup \ldots$ has just been defined. We will show that it meets the requirements.

Suppose that it does not; that is, there are $i<\omega$, a line $\ell$, and distinct $a, b, c \in S_{i} \cap \ell$. Let $\sigma_{i}=\left\langle r_{0}, r_{1}, \ldots, r_{k-1}, r_{k}, j_{0}, j_{1}, \ldots, j_{k-1}, s\right\rangle$. Then $s=$ $\pi(a)=\pi(b)=\pi(c)$. Then, by Theorem 2.1, $\ell \in \lambda(a) \cap \lambda(b) \cap \lambda(c)$. Thus, there is some $p<k$ such that $r_{p}<e(\ell)<r_{p+1}$. But then $a=P_{j_{p}}(\ell)=b$, which is a contradiction.

It is possible to get results for vector spaces whose dimensions exceed $2^{\aleph_{0}}$. For an infinite cardinal $\kappa$ and $n<\omega$, define $\kappa^{+n}$ inductively: $\kappa^{+0}=\kappa$ and $\kappa^{+(n+1)}=\left(\kappa^{+n}\right)^{+}$. The proof of the following theorem uses an inductive argument which has Theorem 2.1 as its starting point.

TheOREM 2.4. Let $m<\omega$. Let $V$ be a vector space over $\mathbb{R}$ such that $\operatorname{dim}(V) \leq\left(2^{\aleph_{0}}\right)^{+m}$. Then there are functions $\pi: V \rightarrow \omega$ and $\lambda: V \rightarrow$ $[\mathcal{L}(V)]^{<\omega}$ such that for any line $\ell \in \mathcal{L}(V)$ and any $X \subseteq \ell$, if $|X| \geq m+3$ and $\pi$ is constant on $X$, then there are at least 3 points $x \in X$ such that $\ell \in \lambda(x)$.

Proof. The proof is by induction on $m$. The case $m=0$ follows from Theorem 2.1. We will now prove the statement for $m+1$ assuming its truth for $m$. Let $\mu=\operatorname{dim}(V)=|V|=\left(2^{\aleph_{0}}\right)^{+(m+1)}$. There is a sequence $\left\langle V_{\alpha}\right.$ : $\alpha<\mu\rangle$, called a filtration of $V$, such that the following hold:

(1) if $\alpha<\mu$, then $V_{\alpha}$ is a subspace of $V$ and $\operatorname{dim}\left(V_{\alpha}\right)<\mu$;

(2) if $\alpha<\beta<\mu$, then $V_{\alpha} \subseteq V_{\beta}$;

(3) if $\gamma<\mu$ is a limit ordinal, then $V_{\gamma}=\bigcup\left\{V_{\alpha}: \alpha<\gamma\right\}$;

(4) $V=\bigcup\left\{V_{\alpha}: \alpha<\mu\right\}$.

For each $\alpha<\mu$, by the inductive hypothesis, let $\pi_{\alpha}: V_{\alpha} \rightarrow \omega$ and $\lambda_{\alpha}: V_{\alpha} \rightarrow\left[\mathcal{L}\left(V_{\alpha}\right)\right]^{<\omega}$ be such that whenever $Y \subseteq \ell \in \mathcal{L}\left(V_{\alpha}\right),|Y| \geq m+2$ and $\pi_{\alpha}$ is constant on $Y$, then there are at least 3 elements $y \in Y$ such that $y \in \ell \in \lambda_{\alpha}(y)$.

Having obtained these sequences, define $\pi$ and $\lambda$ as follows. Consider some $a \in V$ and let $\alpha$ be the least such that $a \in V_{\alpha}$. Then let $\pi(a)=\pi_{\alpha}(a)$ and $\lambda(a)=\lambda_{\alpha}(a)$.

Having defined $\pi$ and $\lambda$, we must show that they work. So suppose not. Then there are a line $\ell$ and $X \subseteq \ell$ contradicting the conclusion of the lemma. Let $\alpha$ be the least ordinal for which $X \subseteq V_{\alpha}$. Clearly, $\alpha \neq 0$ and $\alpha$ is not a limit ordinal. Therefore, $\alpha=\beta+1$ for some $\beta$. Let $Y=X \backslash V_{\beta}$. Clearly $|Y| \geq m+2$, as otherwise we would have $\left|X \cap V_{\beta}\right| \geq 2$, so that $\ell \in \mathcal{L}\left(V_{\beta}\right)$ and thus $X \subseteq V_{\beta}$, contradicting the minimality of $\alpha$. But then $Y$ contradicts the conditions on $\pi_{\alpha}$ and $\lambda_{\alpha}$.

We do not know whether Lemma 2.4 can be strengthened so that the conclusion holds for all $x \in X$ or even for at least 4 points in $X$. 
Corollary 2.5. Let $m<\omega$. Let $V$ be a vector space over $\mathbb{R}$ such that $\operatorname{dim}(V) \leq\left(2^{\aleph_{0}}\right)^{+m}$. Let $\mathcal{L}(V)=L_{0} \cup L_{1} \cup L_{2} \cup \ldots$ be a countable partition. Then there is a countable partition $V=S_{0} \cup S_{1} \cup S_{2} \cup$.. such that whenever $\ell \in L_{i}$, then $\left|\ell \cap S_{i}\right| \leq m+2$.

Pr o of. See remark following the proof of Theorem 0.4.

There is a similar extension of Corollary 2.3.

TheOREM 2.6. Let $m<\omega$. Let $V$ be a vector space over $\mathbb{R}$ such that $\operatorname{dim}(V) \leq\left(2^{\aleph_{0}}\right)^{+m}$. Let $S \subseteq V$ be such that each line of $V$ meets $S$ in a countable set. Then there is a countable partition $S=S_{0} \cup S_{1} \cup S_{2} \cup$.. such that whenever $\ell$ is a line and $i<\omega$, then $\left|\ell \cap S_{i}\right| \leq m+2$.

Proof. The proof is by induction on $m$. The case $m=0$ is just Corollary 2.3. We will prove the theorem for $m+1$ assuming its truth for $m$. Let $\mu=\operatorname{dim}(V)=|V|=\left(2^{\aleph_{0}}\right)^{+(m+1)}$. As in the proof of Theorem 2.4, there is a filtration $\left\langle V_{\alpha}: \alpha<\mu\right\rangle$ of $V$ so that (1)-(4) in that proof hold, and also the following holds:

(5) if $\alpha<\mu$ and $\ell$ is a line of $V$ such that $\left|\ell \cap V_{\alpha} \cap S\right| \geq 2$, then $\ell \cap S \subseteq V_{\alpha+1}$.

For each $a \in V$ we will determine $i<\omega$ such that $a \in S_{i}$.

For each $\alpha<\mu$, by the inductive hypothesis on $m$, there is a partition $S \cap V_{\alpha}=S_{0}^{\alpha} \cup S_{1}^{\alpha} \cup S_{2}^{\alpha} \cup \ldots$ such that whenever $\ell$ is a line of $V$ and $i<\omega$, then $\left|\ell \cap S_{i}^{\alpha}\right| \leq m+2$. Let

$$
S_{i}=S_{i}^{0} \cup \bigcup\left\{S_{i}^{\alpha+1} \backslash S_{i}^{\alpha}: \alpha<\mu\right\} .
$$

It is easily checked that this partition is as required.

We do not know if the bound of $m+2$ in Theorem 2.6 is optimal.

3. Points and hyperplanes. A hyperplane of a vector space is a translate of a subspace. Lines are just 1-dimensional hyperplanes. For any $d<\omega$ and vector space $V$, let $\mathcal{L}_{d}(V)$ be the set of all $d$-dimensional hyperplanes of $V$, and let $\mathcal{L}_{<\omega}(V)$ be the set of all finite-dimensional subspaces of $V$. The aim of this section is to obtain generalizations of the results of $\S 1$ which apply to $\mathcal{L}_{d}(V)$ or $\mathcal{L}_{<\omega}(V)$ instead of just $\mathcal{L}(V)$.

The following theorem generalizes Theorem 2.1.

THEOREM 3.1. Let $V$ be a vector space over $\mathbb{R}$ such that $\operatorname{dim}(V) \leq 2^{\aleph_{0}}$. Then there are functions $\pi: V \rightarrow \omega$ and $\lambda: V \rightarrow[\mathcal{L}(V)]^{<\omega}$ such that whenever $1 \leq d<\omega, H \in \mathcal{L}_{d}(V)$ and $X \subseteq H$ are such that $\pi$ is constant on $X$ and $|X|>2^{d}$, then there are at least 3 points $x \in X$ for which there is $\ell \in \lambda(x)$ such that $x \in \ell \subseteq H$. 
Pr o o f. The functions $\pi$ and $\lambda$ which will do the trick here are exactly the same as the ones that occur in the proof of Theorem 1.2. We will prove that they work, first for finite-dimensional $V$ and then for infinite-dimensional $V$.

Consider finite-dimensional $V$, so let $V=\mathbb{R}^{n}$. We will prove by induction on $d$, where $1 \leq d<n$, that $\pi$ and $\lambda$ have the required property.

If $d=1$, then this follows from Theorem 2.1. So assume that $2 \leq d<n$ and that for all smaller values of $d$ the functions $\pi$ and $\lambda$ have the required property. Let $X$ and $H$ satisfy the hypotheses, and let $Z=\{x \in X: x \in$ $\ell \in \lambda(x)$ for some $\ell \subseteq H\}$. We will show that $|Z| \geq 3$.

Suppose, to the contrary, that $|Z|<3$. Let $a, b \in X \backslash Z$ be distinct. Let $i=\pi(a)$ and let $\sigma_{i}=\left\langle r_{0}, r_{1}, \ldots, r_{k}, j\right\rangle$. Since $a, b$ are distinct, there are $p<k, u \in \operatorname{supp}(a)$ and $v \in \operatorname{supp}(b)$ such that $r_{p}<u, v<r_{p+1}$ and $u \neq v$. Without loss of generality, we can conclude from the pigeon-hole principle that $Y=\{x \in X: u \notin \operatorname{supp}(x)\}$ has more than $2^{d-1}$ elements.

If $\operatorname{Span}(Y) \neq H$, then $1 \leq \operatorname{dim}(\operatorname{Span}(Y))<d$, so by the inductive hypothesis on $d,|Z \cap Y| \geq 3$. Therefore, $\operatorname{Span}(Y)=H$. Then, since $a \notin Z$, the $p$ th tangent line at $a$ meets $H$ at just the point $a$. Let $\theta(x)$ be the $p$ th coordinate function for $a$. Then $a$ is the unique point $x$ (in some definable open neighborhood of $a)$ such that $\theta(x) \in H$, so that $u$ is $(\bigcup\{\operatorname{supp}(x): x \in$ $X\} \backslash\{u\}$ )-definable, which is a contradiction. This completes the proof of the claim and also of the theorem for finite-dimensional $V$.

The extension to infinite-dimensional $V$ is much like the proof of Theorem 2.1. Assume $\operatorname{dim}(V)=2^{\aleph_{0}}$, and let $\left\{v_{x}: x \in \mathbb{R}\right\}$ be a basis for $V$, where $x \neq y \Rightarrow v_{x} \neq v_{y}$. Define naps $(a)$ and $V(X)$ as in the proof of Theorem 2.1. For $Y \in[\mathbb{R}]^{<\omega}$, let $\pi_{Y}: V(Y) \rightarrow \omega$ and $\lambda_{Y}: V(Y) \rightarrow[\mathcal{L}(V(Y))]^{<\omega}$ be such that whenever $H \in \mathcal{L}_{<\omega}(V(Y))$ and $Y \subseteq H$ are such that $\pi_{Y}$ is constant on $Y$ and $|Y|>2^{d}$, then there are at least 3 points $x \in Y$ for which there is $\ell \in \lambda(x)$ such that $x \in \ell \subseteq H$.

We now define $\pi$ and $\lambda$. Consider $a \in V$ and let $Y=\operatorname{naps}(a)$. Then set $\lambda(a)=\lambda_{Y}(a)$. Let $\left\{r_{0}, r_{1}, \ldots, r_{k}\right\}_{<}$be a rational separator for $Y$, and then let $\pi(a)=i$, where $\sigma_{i}=\left\langle r_{0}, r_{1}, \ldots, r_{k}, \pi_{Y}(a)\right\rangle$.

We next show, by induction on $d$, that $\pi$ and $\lambda$ have the required property. For $d=1$, this was done in the proof of Theorem 2.1. So suppose that $d>1$ and that $\pi$ and $\lambda$ have the required property for all smaller values of $d$. Suppose that $X \subseteq H \in \mathcal{L}_{d}(V),|X|>2^{d}$ and $\pi$ is the constant $i$ on $X$. Let $\sigma_{i}=\left\langle r_{0}, r_{1}, \ldots, r_{k}, j\right\rangle$. For a contradiction, suppose that $X$ does not have 3 points as the theorem implies.

If for some $Y$, naps $(a)=Y$ for each $a \in X$, then the 3 points can be found in $V(Y)$. So we can assume that for some $a, b \in X, \operatorname{naps}(a) \neq \operatorname{naps}(b)$. Therefore, without loss of generality, we can assume that there are $p<k$ and $x \in \operatorname{naps}(a)$ such that $r_{p}<x<r_{p+1}$ and $Z=\{c \in X: x \in \operatorname{naps}(a)\}$ has more than $2^{d-1}$ points. If $\operatorname{dim}(\operatorname{Span}(Z))<d$, then the inductive hypothesis 
implies that there are the 3 points in $Z$. However, if $\operatorname{dim}(\operatorname{Span}(Z)) \geq d$, then in fact $\operatorname{Span}(Z)=H$, and this yields a contradiction since $a \in H$ but $x \in \operatorname{naps}(a) \backslash \bigcup\{\operatorname{naps}(c): c \in Z\}$.

The optimality of Theorem 3.1 is open. We do not know if, in general, the number 3 occurring in that theorem can be replaced with some larger number (depending on $d$ ), nor do we know if, in general, the number $2^{d}$ can be replaced with some smaller number.

The next objective is to generalize Theorem 0.4 or even Corollary 2.2. One problem immediately surfaces. Suppose that $\mathcal{L}_{2}\left(\mathbb{R}^{3}\right)=L_{0} \cup L_{1} \cup L_{2} \cup \ldots$ and there is a line $\ell$ such that for each $i<\omega$ there is $H \in L_{i}$ for which $\ell \subseteq H$. Then, for any partition $\mathbb{R}^{3}=S_{0} \cup S_{1} \cup S_{2} \cup \ldots$, there are $i<\omega$ and an $H \in L_{i}$ such that $H \cap S_{i}$ is uncountable. This example led Erdős, Jackson \& Mauldin to make the following definition in [5]. A function $P: \mathcal{L}_{d}(V) \rightarrow[\omega]^{<\omega}$ is said to be acceptable if for each line $\ell$, the set $\bigcup\left\{P(H): \ell \subseteq H \in \mathcal{L}_{d}\left(\mathbb{R}^{n}\right)\right\}$ is finite. The function $P: \mathcal{L}_{<\omega}(V) \rightarrow[\omega]^{<\omega}$ is acceptable if the restrictions to each $\mathcal{L}_{d}(V)$ are acceptable.

The following corollary is a generalization of Corollary 2.2.

Corollary 3.2. Let $V$ be a vector space over $\mathbb{R}$ such that $\operatorname{dim}(V) \leq$ $2^{\aleph_{0}}$, and let $P: \mathcal{L}_{<\omega}(V) \rightarrow[\omega]^{<\omega}$ be acceptable. Then there is a function $Q: V \rightarrow \omega$ such that whenever $1 \leq d<\omega, H \in \mathcal{L}_{d}(V)$, and $i \in H$, then $|\{x \in H: Q(x)=i\}| \leq 2^{d}$.

Proof. For finite-dimensional $V$, this follows from Theorem 3.1 just the way Theorem 0.4 followed from Theorem 2.1. Let $\pi$ and $\lambda$ be as in Theorem 2.1. For given $a \in V$, let $Q(a)=i$, where, for some $s<\omega, \sigma_{i}=\langle\pi(a), s\rangle$ and $i \notin P(H)$ whenever $\ell \subseteq H \in \mathcal{L}_{<\omega}(V)$ and $\ell \in \lambda(a)$.

The infinite-dimensional case follows from the finite-dimensional one in much the same way as was done in Theorems 2.1 and 3.1.

The result in Corollary 3.2 can be pushed up to vector spaces over $\mathbb{R}$ having dimension $\left(2^{\aleph_{0}}\right)^{+m}$ by using Lemma 3.3. Theorem 3.1 has a similar extension using Lemma 3.4 below. Define the function $g^{*}(\kappa, d)$, where $\kappa$ is an infinite cardinal and $d<\omega$, as follows: $g^{*}(\kappa, d)$ is the least $m<\omega$ (if there is one) such that whenever $V$ is a vector space over $\mathbb{R}, \operatorname{dim}(V) \leq \kappa$ and $P: \mathcal{L}_{d}(V) \rightarrow[\omega]^{<\omega}$ is acceptable, then there is a function $Q: V \rightarrow$ $\omega$ such that whenever $H \in \mathcal{L}_{d}(V)$ and $i \in P(H)$, then $\mid\{x \in H: Q(x)$ $=i\} \mid \leq m$. Also define $h^{*}(\kappa, d)$ to be the least $m<\omega$ (if there is one) such that whenever $V$ is a vector space over $\mathbb{R}$ and $\operatorname{dim}(V) \leq \kappa$, then there are functions $\pi: V \rightarrow \omega$ and $\lambda: V \rightarrow[\mathcal{L}(V)]^{<\omega}$ such that whenever $H \in \mathcal{L}_{d}(V)$ and $X \subseteq H$ are such that $\pi$ is constant on $X$ and $|X|>h^{*}(\kappa, d)$, then there are $x \in X$ and $\ell \in \lambda(x)$ such that $x \in \ell \subseteq H$.

Lemma 3.3. $g^{*}\left(\kappa^{+}, d+1\right) \leq g^{*}\left(\kappa^{+}, d\right)+g^{*}(\kappa, d+1)$. 
Lemma 3.4. $h^{*}\left(\kappa^{+}, d+1\right) \leq h^{*}\left(\kappa^{+}, d\right)+h^{*}(\kappa, d+1)$.

Proofs of Lemmas 3.3 and 3.4. Let $V$ be a vector space over $\mathbb{R}$ having dimension $\mu=\kappa^{+}$. Let $\left\langle V_{\alpha}: \alpha<\mu\right\rangle$ be a filtration as in the proof of Lemma 2.4, so that conditions (1)-(4) are satisfied.

For the proof of Lemma 3.4, we let $\pi_{\alpha}$ and $\lambda_{\alpha}$ be demonstrators of the bounds $h^{*}(\kappa, d+1)$. Then define $\pi$ and $\lambda$ on $V$ as follows. Consider $a \in V$ and let $\alpha$ be the least ordinal such that $a \in V_{\alpha}$. Then let $\pi(a)=\pi_{\alpha}(a)$ and $\lambda(a)=\lambda_{\alpha}(a)$. By induction on $d$, we will see that these functions work. The argument for $d=1$ is in Lemma 2.4, and for $d>1$ it is very similar. Suppose $H \in \mathcal{L}_{d}(V)$ and $X \subseteq H$, where $|X|=h^{*}\left(\kappa^{+}, d\right)+h^{*}(\kappa, d+1)+1$, is a counterexample. Thus, $\pi$ is constant on $X$ and for no $x \in X$ and line $\ell \in \lambda(a)$ is $\ell \subseteq H$. Let $\alpha$ be the least ordinal such that $X \subseteq V_{\alpha}$. Clearly, $\alpha \neq 0$ and $\alpha$ is not a limit ordinal, so that $\alpha=\beta+1$ for some $\beta$. Let $Y=X \cap V_{\beta}$. Then either $|Y|>h^{*}\left(\kappa^{+}, d\right)$ or $|X \backslash Y|>h^{*}(\kappa, d+1)$. Suppose the first alternative $|Y|>h^{*}\left(\kappa^{+}, d\right)$. Then by the inductive hypothesis, $\operatorname{dim}(\operatorname{Span}(Y))>d$ so that $\operatorname{Span}(Y)=H$. But then $X \subseteq V_{\beta}$, contradicting the minimality of $\alpha$. Next, suppose the second alternative $|X \backslash Y|>h^{*}(\kappa, d+1)$. But then there are three points $x \in X \backslash Y$ for which there is $\ell \in \lambda_{\alpha}(x)=\lambda(x)$ such that $x \in \ell \subseteq H$. This is also a contradiction, completing the proof of Lemma 3.4.

For the proof of Lemma 3.3, we are given an acceptable $P: V \rightarrow[\omega]^{<\omega}$. Then each $P_{\alpha}=P \mid V_{\alpha}$ is acceptable. Let $Q_{\alpha}: V_{\alpha} \rightarrow \omega$ demonstrate the bounds of $g^{*}(\kappa, d+1)$. Consider $a \in V$. Then let $\alpha$ be the least ordinal such that $a \in V_{\alpha}$ and $Q(a)=Q_{\alpha}(a)$. The proof is now completed in the same way as the proof of the previous paragraph.

Now use the previous two lemmas to push up the dimensions in Theorem 3.1 and Corollary 3.2.

Let the function $k(m, d)$, where $m, d<\omega$, be defined by

$$
k(m, d)=\sum_{i=0}^{d}\left(\begin{array}{c}
m+d \\
i
\end{array}\right) .
$$

Alternatively, we can define $k$ inductively by letting $k(m, 0)=1$ and $k(0, d)$ $=2^{d}$, and then letting

$$
k(m+1, d+1)=k(m, d+1)+k(m, d+1) .
$$

This definition fits in with Lemmas 3.3 and 3.4. The following two corollaries then result.

COROLlary 3.5. Let $m<\omega$ and $V$ be a vector space over $\mathbb{R}$ such that $\operatorname{dim}(V) \leq\left(2^{\aleph_{0}}\right)^{+m}$. Then there are functions $\pi: V \rightarrow \omega$ and $\lambda: V \rightarrow$ $[\mathcal{L}(V)]^{<\omega}$ such that whenever $1 \leq d<\omega, H \in \mathcal{L}_{d}(V)$ and $X \subseteq H$ are such that $\pi$ is constant on $X$ and $|X|>k(m, d)$, then there is $x \in X$ for which there is $\ell \in \lambda(x)$ such that $x \in \ell \subseteq H$. 
COROLlary 3.6. Let $m<\omega$ and $V$ be a vector space over $\mathbb{R}$ such that $\operatorname{dim}(V) \leq\left(2^{\aleph_{0}}\right)^{+m}$, and let $P: \mathcal{L}_{<\omega}(V) \rightarrow[\omega]^{<\omega}$ be acceptable. Then there is a function $Q: V \rightarrow \omega$ such that whenever $1 \leq d<\omega, H \in \mathcal{L}_{d}(V)$, and $i \in H$, then $|\{x \in H: Q(x)=i\}| \leq k(m, d)$.

We do not know how large the dimension of the vector spaces in either of those corollaries can be and still get some finite bounds.

Corollary 3.2 shows the existence of a function $g: \omega \rightarrow \omega$ defined as follows: If $d<\omega$, then $g(d)$ is the least $m<\omega$ such that whenever $n<\omega$ and $P: \mathcal{L}_{d}\left(\mathbb{R}^{n}\right) \rightarrow[\omega]^{<\omega}$ is acceptable, then there is a function $Q: \mathbb{R}^{n} \rightarrow \omega$ such that whenever $H \in \mathcal{L}_{d}\left(\mathbb{R}^{n}\right)$ and $i \in P(H)$, then $|\{x \in H: Q(x)=i\}| \leq m$. Indeed, Corollary 3.2 asserts that $g(d) \leq 2^{d}$.

There is another bound for $g(d)$ involving binomial coefficients proved by Erdős, Jackson \& Mauldin [5]:

$$
2^{\aleph_{0}} \leq \aleph_{m} \Rightarrow g(d) \leq\left(\begin{array}{c}
m+d \\
d
\end{array}\right) .
$$

This result is incomparable with Corollary 3.2. For example, Corollary 3.2 asserts that $g(2) \leq 4$ and $g(3) \leq 8$, and, on the other hand, the result in [5] asserts that $2^{\aleph_{0}}=\aleph_{2} \Rightarrow g(2) \leq 6$ and $2^{\aleph_{0}}=\aleph_{1} \Rightarrow g(3) \leq 4$. By combining Corollary 3.2 with the inductive proof of the result in [5], we can get simultaneous improvements to both results.

The next lemma captures the inductive step of the result in [5]. We refine the function $g$ to be a function of two variables: an infinite cardinal $\kappa \leq 2^{\aleph_{0}}$ and $d<\omega$. Let $g(\kappa, d)$ be the least $m<\omega$ such that whenever $n<\omega, F$ is a subfield of $\mathbb{R}$ such that $|F| \leq \kappa$, and $P: \mathcal{L}_{d}\left(F^{n}\right) \rightarrow[\omega]^{<\omega}$ is acceptable, then there is a function $Q: F^{n} \rightarrow \omega$ such that whenever $H \in \mathcal{L}_{d}\left(F^{n}\right)$ and $i \in P(H)$, then $|\{x \in H: Q(x)=i\}| \leq m$. Of course, $g\left(\aleph_{0}, d\right)=1$ and $g(d)=g\left(2^{\aleph_{0}}, d\right)$, so this lemma yields the bound in $(*)$.

Lemma $3.7([5]) \cdot g\left(\kappa^{+}, d+1\right) \leq g\left(\kappa^{+}, d\right)+g(\kappa, d+1)$.

Thus, whereas Corollary 3.2 asserts that $g(4) \leq 16$ and the result in [5] implies that $2^{\aleph_{0}}=\aleph_{2} \Rightarrow g(4) \leq 15$, the proofs of the two combine to yield that $2^{\aleph_{0}}=\aleph_{2} \Rightarrow g(4) \leq 13$. However, we can do even better; a corollary of the next theorem improves the result in [5] and shows, in particular, that $2^{\aleph_{0}}=\aleph_{2} \Rightarrow g(4) \leq 9$.

Define the function $h: \omega \times \omega \rightarrow \omega$ as follows. Given $d, m<\omega$, let $q<\omega$ and $r<m$ be such that $d=q m+r$. Then set

$$
h(m, d)=(q+2)^{r}(q+1)^{m-r} .
$$

It follows that

$$
\frac{h(m, d+1)}{h(m, d+1)-h(m, d)}=\left[\frac{d}{m}\right]+2
$$


when $m>0$. Alternatively, we can define $h$ inductively by letting $h(m, 0)=$ $h(0, d)=1$, and then letting

$$
h(m, d+1)=\frac{[d / m]+2}{[d / m]+1} h(m, d)
$$

when $m>0$. It is easily checked that

$$
h(m, d) \leq 2^{d} \quad \text { and } \quad h(m, d) \leq\left(\begin{array}{c}
m+d \\
d
\end{array}\right) .
$$

THEOREM 3.8. (Assume $2^{\aleph_{0}} \leq \aleph_{m}$.) There are functions $\pi: \mathbb{R}^{n} \rightarrow \omega$ and $\lambda: \mathbb{R}^{n} \rightarrow\left[\mathcal{L}\left(\mathbb{R}^{n}\right)\right]^{<\omega}$ such that whenever $1 \leq d<n, H \in \mathcal{L}_{d}\left(\mathbb{R}^{n}\right)$ and $X \subseteq H$ are such that $\pi$ is constant on $X$ and $|X|>h(m, d)$, then there are at least 3 points $x \in X$ for which there is $\ell \in \lambda(x)$ such that $x \in \ell \subseteq H$.

We first present a couple of known lemmas.

LEMma 3.8.1. Let $m<\omega$. There are functions $F:\left[\omega_{m}\right]^{m+1} \rightarrow \omega$ and $f:\left[\omega_{m}\right]^{m+1} \rightarrow \omega_{m}$ such that:

(1) if $A \in\left[\omega_{m}\right]^{m+1}$, then $f(A) \in A$;

(2) if $A, B \in\left[\omega_{m}\right]^{m+1}, F(A)=F(B)$ and $i \leq m$, then $f(A)$ is the ith element of $A$ iff $f(B)$ is the ith element of $B$;

(3) if $A, B \in\left[\omega_{m}\right]^{m+1}, F(A)=F(B)$ and $A \neq B$, then $A \backslash\{f(A)\} \neq$ $B \backslash\{f(B)\}$.

Proof. The proof is by induction on $m$. If $m=0$, then the result is trivial: let $F(\{n\})=f(\{n\})=n$. Assume that we have $F^{\prime}:\left[\omega_{m}\right]^{m+1} \rightarrow \omega$ and $f^{\prime}:\left[\omega_{m}\right]^{m+1} \rightarrow \omega_{m}$ which work for $\omega_{m}$. We define $F$ and $f$ for $\omega_{m+1}$. For each $\alpha<\omega_{m+1}$, let $g_{\alpha}: \alpha \rightarrow \omega_{m}$ be an injection. Now consider some $A \in\left[\omega_{m+1}\right]^{m+2}$. Let $\alpha=\max (A)$ and let $B=\left\{g_{\alpha}(\beta): \beta \in \alpha \cap A\right\}$. Let $F(A)=F^{\prime}(B)$ and let $f(A)=g_{\alpha}^{-1} f^{\prime}(B)$. It is easily checked that $F$ and $f$ are as required.

Lemma 3.8.2. (Assume $2^{\aleph_{0}}=\aleph_{m}$.) There are functions $F:[\mathbb{R}]^{m+1} \rightarrow \omega$ and $f:[\mathbb{R}]^{m+1} \rightarrow \mathbb{R}$ such that:

(1) if $A \in[\mathbb{R}]^{m+1}$, then $f(A) \in A$;

(2) if $A, B \in[\mathbb{R}]^{m+1}, F(A)=F(B)$ and $i \leq m$, then $f(A)$ is the ith element of $A$ iff $f(B)$ is the ith element of $B$;

(3) if $A, B \in[\mathbb{R}]^{m+1}, F(A)=F(B)$ and $A \neq B$, then $A \backslash\{f(A)\} \neq$ $B \backslash\{f(B)\} ;$

(4) if $A=\left\{a_{0}, a_{1}, \ldots, a_{m}\right\}_{<} \in[\mathbb{R}]^{m+1}$, then there is a rational separator $R=\left\{r_{0}, r_{1}, \ldots, r_{m+1}\right\}$ of $A$ such that whenever $B \in[\mathbb{R}]^{m+1}$ and $F(A)=$ $F(B)$, then $R$ is a rational separator for $B$.

Proof. Let $F^{\prime}$ and $f^{\prime}$ be functions as in Lemma 3.8.1 for $\omega_{m}$. Let $g: \mathbb{R} \rightarrow$ $\omega_{m}$ be a bijection. We now define $F$ and $f$. Consider $A \in[\mathbb{R}]^{m+1}$. Let $f(A)=$ 
$g^{-1} f^{\prime} g(A)$. To define $F(A)$, let $A=\left\{a_{0}, a_{1}, \ldots, a_{m}\right\}_{<}$and let $s_{0}, s_{1}, \ldots, s_{m}$ be such that $g\left(a_{s_{0}}\right)<g\left(a_{s_{1}}\right)<\ldots<g\left(a_{s_{m}}\right)$. Let $\left\{r_{0}, r_{1}, \ldots, r_{m+1}\right\}<$ be a rational separator for $A$. Finally, let $F(A)=i$ where

$$
\sigma_{i}=\left\langle F^{\prime}(g(A)), r_{0}, r_{1}, \ldots, r_{m+1}, s_{0}, s_{1}, \ldots, s_{m}\right\rangle .
$$

It is easily checked that $F$ and $f$ work.

Proof of Theorem 3.8. It suffices to prove that if $1 \leq d<n$ then there are functions $\pi_{d}: \mathbb{R}^{n} \rightarrow \omega$ and $\lambda_{d}: \mathbb{R}^{n} \rightarrow\left[\mathcal{L}\left(\mathbb{R}^{n}\right)\right]^{<\omega}$ such that whenever $H \in$ $\mathcal{L}_{d}\left(\mathbb{R}^{n}\right)$ and $X \subseteq H$ are such that $\pi_{d}$ is constant on $X$ and $|X|>h(m, d)$, then there are at least 3 elements $x \in X$ for which there is $\ell \in \lambda_{d}(x)$ such that $x \in \ell \subseteq H$. We will prove the existence of $\pi_{d}$ and $\lambda_{d}$ by induction on $d$. Let $\pi_{1}$ and $\lambda_{1}$ be the functions from Theorem 2.1 or Theorem 3.1. Now suppose that $1<d<n$ and that we have $\pi_{k}$ and $\lambda_{k}$ for $1 \leq k<d$. We will obtain $\pi_{d}$ and $\lambda_{d}$.

Let $\lambda_{d}=\lambda_{d-1}$. To get $\pi_{d}$, let $F$ and $f$ be as in Lemma 3.8.2. Let $\pi_{d}$ be such that the following holds.

Suppose $a, b \in \mathbb{R}^{n}$ are such that $a=b$. Then $\pi_{d-1}(a)=\pi_{d-1}(b)$ and $\pi_{1}(a)=\pi_{1}(b)$. Also $|\operatorname{supp}(a)|=|\operatorname{supp}(b)|$, so there is a unique orderpreserving bijection $e: \operatorname{supp}(a) \rightarrow \operatorname{supp}(b)$. Then, if $A \in[\operatorname{supp}(a)]^{m+1}$, then $F(A)=F(e(A))$.

We show that $\pi_{d}$ and $\lambda_{d}$ work. Let $X$ and $H$ satisfy the hypotheses, and let $Z=\left\{x \in X: x \in \ell \in \lambda_{d}(x)\right.$ for some $\left.\ell \subseteq H\right\}$. We will show that $|Z| \geq 3$ by assuming the contrary. For some (or all) $a \in X$, let $i=\pi_{1}(a)$ and let $\sigma_{i}=\left\langle r_{0}, r_{1}, \ldots, r_{k}, j\right\rangle$.

Claim 1. If $a \in X$ and $u \in \operatorname{supp}(a)$, then $|\{x \in X: u \notin \operatorname{supp}(x)\}| \leq$ $h(m, d-1)$.

To prove the claim, suppose to the contrary that there are $a$ and $u \in$ $\operatorname{supp}(a)$ such that $|\{x \in X: u \notin \operatorname{supp}(x)\}|>h(m, d-1)$. Let $Y=\{x \in X$ : $u \notin \operatorname{supp}(x)\}$. Then $|Y|>h(m, d-1)$. By the inductive hypothesis on $d$, if $\operatorname{dim}(\operatorname{Span}(Y))<d$, then the claimed three points can be found in $Y$, so we can assume that $\operatorname{Span}(Y)=H$. Let $p<k$ be such that $r_{k}<u<r_{k+1}$ and let $\ell$ be the $p$ th tangent line for $\varphi_{j}$ at $a$. Thus, $\ell \in \lambda_{d}(a)$ and, hence, $\ell \nsubseteq H$. Therefore, $a$ is the unique point of intersection of $\ell$ and $H$. But then $u$ is $(\bigcup\{\operatorname{supp}(x): x \in X\} \backslash\{u\})$-definable, which is a contradiction. The claim is proved.

For each $p<k$ and $x \in X$, let $\langle x\rangle_{p}$ be the $p$ th element in $\operatorname{supp}(x)$. Then let $E_{p}$ be the equivalence relation on $X$ such that $x E_{p} y$ iff $\langle x\rangle_{p}=\langle y\rangle_{p}$. Clearly, the intersection of all these equivalence relations is the equality relation. Let $I \subseteq k$ be a minimal set such that $\bigcap\left\{E_{p}: p \in I\right\}$ is the equality relation.

Claim 2. $|I|>m$. 
By Claim 1, each equivalence class of each $E_{p}$ has at least $|X|-h(m$, $d-1)$ elements. Since $|X|>h(m, d)$, each equivalence class has at least $1+h(m, d)-h(m, d-1)$ elements, so there must be at most $[(1+h(m, d)) /(1+$ $h(m, d)-h(m, d-1))]=[(d-1) / m]+1$ equivalence classes. It then follows from equation $(* *)$ that there are at most $[(d-1) m]+1$ equivalence classes. Since the intersection of all the equivalence relations $E_{p}$, where $p \in I$, is the equality relation, it follows that $([(d-1) / m]+1)^{|I|} \geq h(m, d)$. But, clearly, $([(d-1) / m]+1)^{m}<h(m, d)$, so that $|I|>m$, proving the claim.

Now let $A \subseteq I$ be such that $|A|=m+1$. Because of the minimality of $I$, there are $a, b \in A$ such that $\langle a\rangle_{p}=\langle b\rangle_{p}$ whenever $f(A) \neq p \in A$, and also that $\langle a\rangle_{f(A)} \neq\langle b\rangle_{f(A)}$. But this contradicts Lemma 3.8.2, so the theorem is proved.

Corollary 3.9. If $2^{\aleph_{0}} \leq \aleph_{m}$, then $g(d) \leq h(m, d)$.

4. Questions. There are several unanswered questions which naturally arise from the results of this paper and have to do with the optimality of these results. In some cases, the questions can be interpreted absolutely, and in others in terms of relative consistency. We mention specifically the bounds that occur in Theorem 2.4, Corollary 2.5 and Theorem 2.6, in Theorem 3.1 and Corollary 3.2, in Corollaries 3.5 and 3.6, and in Theorem 3.8 and Corollary 3.9.

\section{References}

[1] R. O. Davies, On a problem of Erdös concerning decompositions of the plane, Proc. Cambridge Philos. Soc. 59 (1963), 33-36.

[2] —, On a denumerable partition problem of Erdös, ibid., 501-502.

[3] P. Erdös, Some remarks on set theory IV, Michigan Math. J. 2 (1953-54), 169-173.

[4] P. Erdös, S. Jackson and R. D. Mauldin, On partitions of lines and space, Fund. Math. 145 (1994), 101-119.

[5] -, 一, -, On infinite partitions of lines and space, ibid. 152 (1997), 75-95.

[6] J. H. Schmerl, Countable partitions of Euclidean space, Math. Proc. Cambridge Philos. Soc. 120 (1996), 7-12.

[7] W. Sierpiński, Sur un théorème équivalent à l'hypothèse du continu $\left(2^{\aleph_{0}}=\aleph_{1}\right)$, Bull. Internat. Acad. Polon. Sci. Lett. Cl. Sci. Math. Nat. Sér. A Sci. Math. 1919, $1-3$.

[8] J. C. Simms, Sierpiński's Theorem, Simon Stevin 65 (1991), 69-163.

Department of Mathematics

University of Connecticut

Storrs, Connecticut 06269, U.S.A.

E-mail: schmerl@math.uconn.edu 